\title{
Study On The Graphic Structure And The Communication Ways Of Chinese Characters In The Light Of New Media
}

\author{
Lin Liu ${ }^{1}$, Wenjun Jiang ${ }^{2}$ \\ ${ }^{1}$ School Of Art Design, Hubei University Of Technology, Wuhan, Hubei Province, China \\ ${ }^{2}$ School Of Art Design, Hubei University Of Technology, Wuhan, Hubei Province, China
}

\begin{abstract}
Objective The new media has promoted the presentation of three-dimensional dynamic Chinese character images on the screen, with temporal and spacial, virtual and interactive characteristics. Therefore, this article aims to study the influence of new media art on the graphic structure and the communication ways of Chinese characters. Method Through the Exploration of the graphic structure of Chinese character and screen, visual representations of the character image and communication ways of the image, this article makes it clear that the character image supported by the new media technology is expanded from two-dimensional to three-dimensional mode, from static to dynamic state. Conclusions There appears more and more three-dimensional character image design which is presented with the new media. For example, animation and hypertext technology add the concept of motion to Chinese character, which means characters are designed in a dynamic way, getting rid of the traditional static subtitle presentation and bringing character design unprecedented opportunities and challenges.
\end{abstract}

\section{Introduction}

Whenever there is a new era, there will be new visual symbols. For this point, the emergence of characters is no exception. For example, Song typeface was created to meet the needs of a large number of printing and reading; boldface was created to enrich the expression forms of newspaper titles and text advertisements at that time; the creation of computer fonts was entirely to meet the needs of computer typesetting and printing. Then, the appearance of Chinese character image is a media symbol design for TV and network screen. Chinese character image has unique performance advantages. Through the display of the screen, the image of Chinese characters not only makes the meaning of the text specific and intuitive, but also creates a new "intertextuality" between the image and the text, thus adding new interest to the art of Chinese characters.

In the late1990s, the informatization and networking of the new digital media made it very easy to spread and duplicate images. People can watch pictures, listen to something, play games and release or receive information on the internet. The development of the image marketing has been speeded up in the visual screen era. The design of Chinese characters has changed from the original paper medium to the visual screen medium, and the relationship between the design generation forms and technologies has been closer. The character image presented in the screen is directly perceived, vividly displayed, and combined with sound. Digital information processing and generation technology (computer graphics, image, digital audio processing technology) plays a pivotal role in the production of dynamic text design. In addition to form, dynamic text also includes 3D modeling, lighting colors, movement patterns, and sound changes. It is widely used in title design, web design and game design. The dynamic text design is flexible and interesting, easy to attract people's attention and enhance memory. The image stream shown in the character design pictoialization has become a new feature of Chinese character image in the new media. For example, different styles of fronts has appeared - some are broken; others like flames or waves, which are not only multidimensional, but also animated, flowing, presented with motioning, musical, virtual character image mode. This chapter discusses the question from three aspects - the graphic structure of Chinese character and screen, visual representations of the character image and communication ways of the image.

\section{The Graphic Structure Of Chinese Character And Screen}

In the early days, the hand-made images were made with brushes, knives and other tools, while the images were original hand-made works such as oil painting, seal cutting and paper cutting, which also made the creation cycle of such images long and the communication channels more restricted. But the modern reproduction technology image is opposite. It has the characteristics of fast generation, and it can be infinitely copied, so this image reproduction technology is most favored by the mass media. For example, for computer image design, images are shooting materials and hand-painted materials 
that are made by machine and created by human brain. In the later stage, new images are formed by combining imaginary pictures with computer. Like the three forms of media, the survival modes of images in the three periods have evolved with the progress of human technology, and they still exist today.

By the end of 1980s, computer font and art font were produced by hand drawing (conception) and computer, and the font form was changed from two-dimensional effect to three-dimensional effect. Since then, the computer, as a tool, not only makes the font design into two image storage modes: vector image and bitmap, but also makes the scale, size, bending and other forms of font design become accurate and standardized. Due to the rapid survival of fonts, the production of works is convenient and fast, and it can be infinitely copied, and its transmission form has also been converted to video mode. The image of Chinese characters is produced by the combination of computer special effects technology and human brain creation. For example, through the use of computers to break up Chinese characters and reconstruct them to form pseudo images with multidimensional space, or to combine the strokes of Chinese characters with virtual imaginary pictures to produce a more complex visual information symbol. The survival mode of its font has also been transformed into the image mode. The main way of expression is to present the title advertisement or virtual image on the screen, which makes it intuitive, vivid, lifelike and pays equal attention to pictures and sound. Among them, light effect and motion are the important factors to form Chinese character design image.

The screen media is displayed in light colors, while the prints are displayed in printing or ink color. For example, paper printing is presented in black on a white background, the text above which is a collection of monochrome dots of black ink, while text on an electronic screen is displayed in pure black, and the resolution constraints or the flowing image patterns causes differences in text clarity. For example, the fixed scale of the TV screen is 4:3 and 16:9 (display models vary). Most screens are larger in width than height, and this fixed scale is a picture presented by a static rectangular frame. The motion of an object in twodimensional space allows the viewer to feel the infinite space outside the screen, while the three-dimensional space make them feel the depth of the screen space, that is to say, the limited screen presents infinite space.

Because the space displayed on the screen is inconsistent with the size of objects in the real world, objects closer to the screen take up more of the image space visually than objects farther away. On the screen, the visual sense of large-area objects is much greater than that of small-area objects. You can resize text by lengthening the lens so that it's readable. Any design is based on a certain amount of space. Plane media is based on two-dimensional flat space, while network media is constructed by two-dimensional and three-dimensional space, in which the text is interspersed. When text and graphics appear on screens made up of three-dimensional virtual space, a spatial system consisting of direction vectors is added compared to the two-dimensional of traditional paper media. As Peter Laurenfield describes in The Reader of the New Medium: "Computers have revived the power of writing and made it popular, and the digital text of letters has risen from its own ashes. A resurgent digital phoenix flies on a monitor, travels through the web, and is active in the visual space." $\mathrm{A}$ computer screen is a window for human interaction with a machine, and all visualizations are presented on the screen. Portable electronic products have generally small screens, with relatively low resolution and very valuable display space. A computer screenshot is a detail of human-computer interaction, which saves the computer screen in the form of text and pictures. Screen is more than the carrier of visual presentation. Its tactile function also enhances the communication between the user and the machine, so that people can truly touch the machine and get the real experience. When people watch the fairyland on TV, they are in a relaxed world, playing a kind of "game" 2 ... This can not be separated from light and color, two-dimensional space, three-dimensional space, time and motion, sound, which are also part of the discussion of the formation of Chinese character images.

Due to the time and mobility of dynamic text, as well as the visual video image and network interaction experience of new media technology, dynamic text design has been applied. Animation key-frames are the beginning and end of an action, while intermediate frames are used to control the rhythm and speed of text in motion. As it shown in Figure 1, the intermediate frame has the function of adjusting and changing the speed of the font in the process of movement, and can produce the movement effect of moving rhythm from fast to slow or from slow to fast. For example, the dynamic text played on the screen is displayed at the speed of 24 frames for per second. Due to the movement change of the text itself, as well as the change of the length of time or sequence, the short-term visual dynamic is caused.

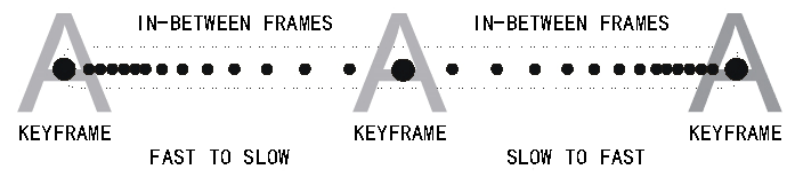

As it shown in Figure 1, the effect of the frame on the font speed and the adjustment effect of text movement mainly depends on the direction and speed. The movement direction shows the movement track of the text. The speed of text movement brings people different psychological feelings. The slow movement is quiet and gentle. The dynamic design of the text presented on the screen, in addition to the emotional infection of the rhythm through the use of key-frames, can also use 3D animation software to design and generate irregular font rotation, supplemented by its different modeling dynamics. Another example is the use of 3D animation software to design and create animated fonts. The movement state, speed and trajectory of the text are very free and irregular, especially the integration of new media and network media interaction platform provides more dynamic performance for text design. More creative space also promotes the innovation of Chinese animation fonts. 


\section{Visual Representations Of The Character Images}

As a visual form of new media art, Chinese character image follows the same basic laws with static character design visual art, such as font and text, scene layout, light, rotation, the picture processing of threedimensional space, but to a large extent it can not be separated from font, light and color, sound and other visual forms. In the interaction, there is a "field" in the process of viewing. Visual image, human perception, and inner emotion are all elements of this field (the field includes text, pictures, colors, light and dynamics, music). Chinese character image mainly depends on the role of this "field" between the elements, which is very similar to the physical force. Images can be seen as "field" consisting of visual elements. If the forces present in the image can cancel each other out, the visual perception will also be balanced ${ }^{3}$. In Figure 1, the poster for the channel of "jinwanwodangjia" ("I'm the leader of my home tonight" in English), contains background graphics that create a dynamic effect on the screen, as well as color elements from dark blue to light color. In the process of forming the Chinese character image, the shape, direction and position of these elements are all around the theme to guide the line of sight, so that the visual elements of the screen are mutually supportive and offset, and form a balanced beauty. Another example is Hunan Satellite TV Variety Channel, "feichangkaopu" (Very Reliable in English) in Figure 2. The font on the red footprint graphic is white imitation Song-Dynastystyle typeface, which is prominent, and the picture is simple and clear, highlighting the footprints that is to be sought; The film Curiosity Kills the Cat (the name of the network propaganda film), uses the image of "cat" subtly formed by Chinese characters and lines with simple color, and the composition of the film's text visualization has a magical meaning.

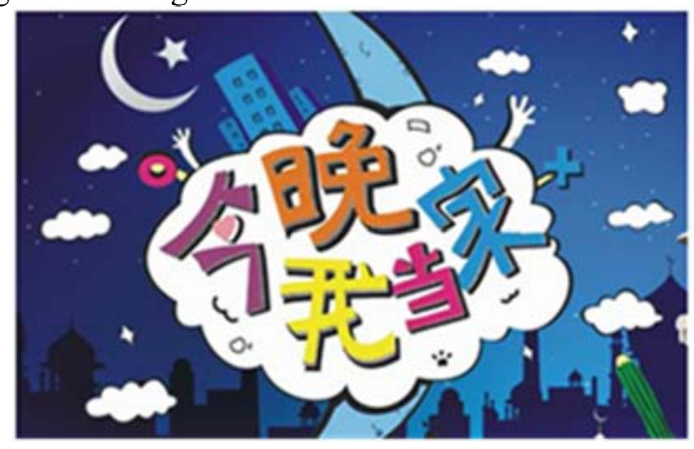

Figure 1 The poster design for the channel of "jinwanwodangjia" (I'm the leader of my home tonigh)

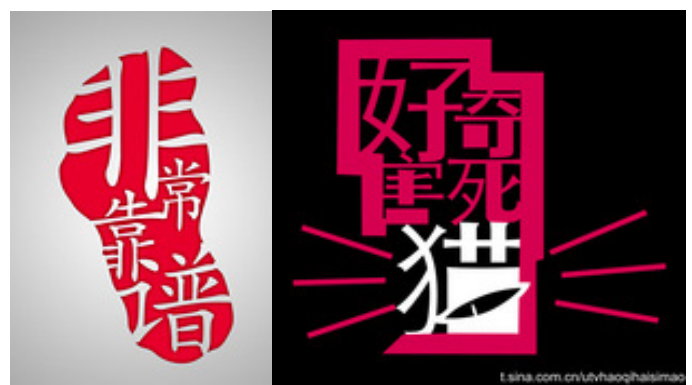

Figure 2 The poster of "feichangkaopu" and "haoqixinhaisimao" (Curiosity Kills the Cat)

Color is as important as other factors in visual form. The dissemination of images by color has become an important factor for the audience to gain visual beauty. In terms of the principles of Gestalt Psychology, the first one, namely the vision, conducts unified treatment of elements with similarities, which can be the similarity in color. In Figure 3, the poster of "tianshengwocai" (a finance and economics program), uses the design of complementary colors, contrasting colors, adjacent colors. The left image is a purple background and yellow text to form a complement color, while the right image is the yellow background and text and blue cube to form a contrast. The color matching gives the viewer a strong aesthetic visual effect.

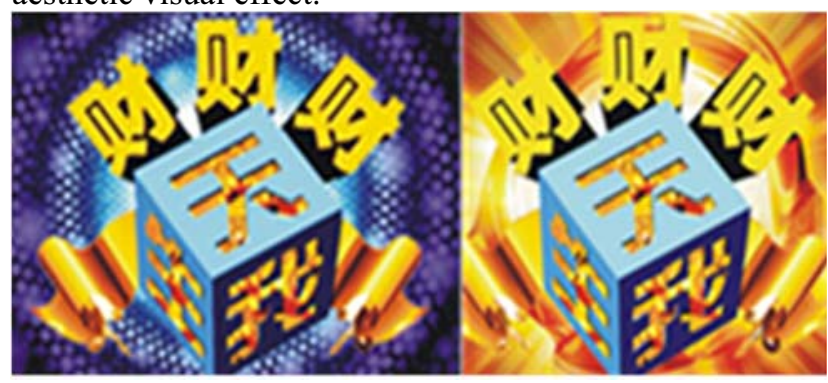

Figure 3 The poster of "tianshengwocai"

The golden division law is used to describe the ratio between parts and the whole. For example, divide a line with a length of 1 into two, of which the longer part is 0.618 and the shorter is 0.382 . The scale is the most comfortable for the visual perception of the composition. The division arrangement design of "Mango Large Live" in 2012 Hunan Satellite TV large-scale live event video column follows the principle of golden division law in regards to its main text position, graphics and background. Its theme font image of "mango large live" is in the short part of the whole position. The art modeling of the text is very interesting, the visual effect of the image is strong, so this visual expression with beauty is more impressive for the audience. As a form of historical proportion, golden law is permeated by the conscious and unconscious pursuit of artists, which is tantamount to a meaningful "inner scale" condensation. The "inner scale" here refers to the inner psychological style of man. In fact, the acceptance of the golden division scale pattern reflects people's interest in visual forms that conform to the laws of visual composition ${ }^{4}$.

The Chinese character image is not simply a stack of pictures, but a comprehensive art. Different from the 
single paper media, it is composed of light and color, two-dimensional and three-dimensional space, time and motion, sound, reality and virtual, and its transmission forms and ways are different. The representative scholar of phenomenology, E. Hussel, analyzed the construction process of image consciousness when discussing image consciousness. E. Hussel believed that an image is not a representation that can be understood simply by perception. In fact, it is constructed by superimposing many essentially different meanings on each other and constructing them in mutual connotation. Therefore, its structure is more complicated. When reading, readers will reveal their different understandings according to the changes and differences of their attention and hobbies, which also shows that looking at images to obtain information is completely different from directly obtaining information from real scenes. For example, for network images and video images, they have direct operability, and the audience can participate in and control image information sharing through web pages. For example, images composed of Chinese characters, city, road and automobile visual elements can show traffic related information; while images composed of folk festival elements and Chinese characters can show traditional national art features, thus making up for the shortcomings of using graphics or simple words as the way of information transmission.

\section{The Communication Ways Of Chinese Character Images}

The light in the Chinese character image of the video belongs to the artificial light, which is controlled with computer synthesis technology, thus producing the effect that the picture or the title needs. Through the light projection and the change of light direction, the strength of color and the depth of space can be changed to enhance the artistic effect of the picture and scene. The aspect ratio is the ratio of the width and height of the screen. Generally speaking, the screen ratio is $4: 3$. afterwards there appeared widescreen movie mode 1.85:1, panoramic visual mode 2.35:1. and HDTV mode 16:9. These different formats of screens have different visual advantages. The larger the screen is, the more powerful visual stimulation can be brought to the viewer, and it is more conducive to the performance of spectacular and beautiful pictures. The visual presentation of two-dimensional space is a balanced picture of text, graphics, color and other elements combined into mutual offsetting of a force. Because Chinese character image is not narrated in a static way, but from one picture to another, that is, the picture image is constantly changing, it needs to consider the visual elements of the picture placed in motion, and to maintain consistency to show a balanced beauty in the motion.

The core of presenting the three-dimensional world in the two-dimensional plane is to use the lens's ability to show the space to embody the three-dimensional sense. "To understand three-dimensional presentation, you need to know a film and television noun 'Z-axis' first. Understanding the 'Z-axis' requires the knowledge of plane geometry. In a two-dimensional plane, determine the vertical and horizontal coordinates, i.e. the $\mathrm{X}$ and $\mathrm{Y}$ values, for any point in the screen. In a three-dimensional space, the $Z$ value can be used to represent the depth of space at this point. Therefore, the Z-axis refers to how far an object appears to be from the camera. This axis can extend indefinitely, not only to the horizon, but also to the audience. The feeling of three-dimensional space is built on the $\mathrm{Z}$ axis ${ }^{5}$. For example, through the relative size of the screen, linear perspective and light strength, the screen can reflect the feeling of three-dimensional space. In figure 4 of the title design of the Yuanying image studio, the continuous text "yuanying", text size, light integration, three-dimensional changes in the text, push and pull processing of close and distant shot, have not only a three-dimensional effect but also the time and movement effect of the four-dimensional space.

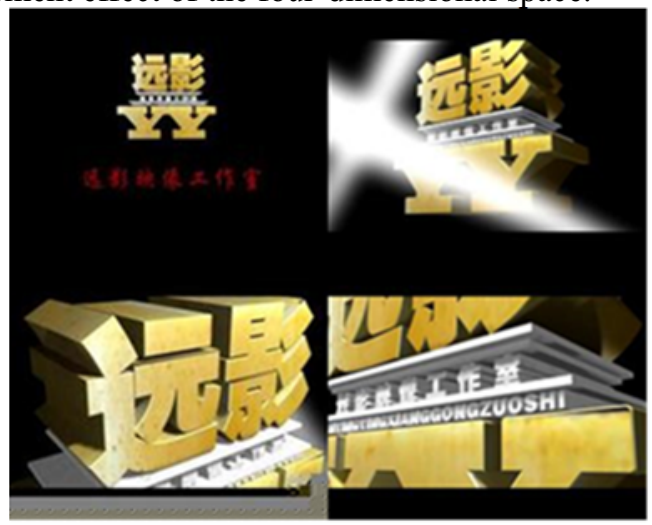

Figure 4 Title design of the Yuanying image studio

The time in the film and television picture can be divided into subjective time and objective time. Subjective time is the viewer's psychological feeling about time. If title of the column has a fast pace, the viewer will have the psychological feeling that time accelerates. Objective time can be considered as the time when web page ads exist. The motion in film and television can be divided into basic, secondary and threelevel motion. The basic motion is the motion of the object itself in the picture, the secondary motion is that of the camera and the motion caused by the push and pull of the lens, and the three-level motion is formed by the switching between the pictures ${ }^{6}$. The difference between Chinese character pictorialization and general design is that Chinese character images constantly create illusions through the use of continuous images, which make people feel that the text and graphics, light and color in the screen are in motion, and most importantly, pass on messages through the movement of the screen.

Sound has the function of expressing information, and the sound itself can transmit information. The content and the strength of the sound can express different meanings, so the combination of sound and picture can make a more complete three-dimensional space. For example, sound can reflect the state of different spaces: fast-paced sound indicates a sense of urgency of time, mixed dubbing and singing sounds are in a KTV room, the sound of flutes and birds is in the wild, horns and jingling sounds are on the road; Sound can also reflect different times: alarm 
sounds in the early morning, bells for class, news broadcast television, etc. can make people feel the time of the sound. By complementing with hearing, we can create a four-dimensional visual environment and surrounding sound, giving people a feeling of movement.

\section{Conclusions}

The image of Chinese characters is produced by the combination of computer special effects technology and human brain creation. For example, through the use of computers to break up Chinese characters and reconstruct them to form pseudo images with multi-dimensional space, or to combine the strokes of Chinese characters with virtual imaginary pictures to produce a more complex visual information symbol. The survival mode of its font has also been transformed into the image mode. The main way of expression is to present the title advertisement or virtual image on the screen, which makes it intuitive, vivid, lifelike and pays equal attention to pictures and sound. Among them, light effect and motion are the important factors to form Chinese character design image. As a form of media, Chinese character images presents in the virtual way of images, and is perceived through the audio, visual and touch senses. The text that people feel is not something that is simply sensory perception, but a synthesis of sensory and psychological experiences. It is not a pure experience of a certain feeling, but a whole-hearted immersion in it. In this multi-dimensional space, it can not only spread information, but also enable the viewer to obtain fresh taste of aesthetic enjoyment. "Compared to the one-way communication of modern media, the interactive communication of new media connects beauty producers and consumers more closely, making both more involved in aesthetic experiences, and even more difficult to separate from each other. Such aesthetics are not static and meditative, nor one-way, but pluralistic and dynamic, endless and open." The image-based presentation of the Chinese character design which expresses its meaning with form and transmit its emotion with meaning, has interpreted the beauty of the Chinese character's form.

\section{REFERENCES}

1. Peter Laurenfeld: The Readers of New Media [M] Cambridge MT Press 2003, p. 691.

2. Ren Yue: Introduction to Visual Communication [M], Renmin University of China Press, 2008, pp. 143144.

3. [U.S.] Rudolf Arnheim: Art and Visual Perception [M], translated by Teng Shou-yao, China Social Science Press, 1984, p. 5.

4. Cao Hui: The Aesthetic Study of Visual Forms [M], People's Publishing House, 2009 edition, p. 180.

5. [U.S.] Herbert Zettel: Image, Sound, Motion: the Aesthetics of Practical Media [M].Translated by Zhao Sensen, Communication University of China Press, 2003 edition, p. 143.
6. Ren Yue: Introduction to Visual Communication [M], Renmin University of China Press, 2008 edition, p. 166.

7. Liu Zili: The Aesthetic Reflections Brought by New Media. (in Literature and History), 2004(5). 